\title{
Location-based effect of decomposition on laminated curved plate structures
}

\section{Can Gonenli ${ }^{a^{*}}\left(\mathbb{D}\right.$, Oguzhan Das ${ }^{b}$}

${ }^{a}$ Department of Machine Drawing and Construction, Ege University, Izmir, Turkey. E-mail: can.gonenli@ege.edu.tr

b Department of Motor Vehicles and Transportation Technologies, Dokuz Eylul University, Izmir, Turkey. E-mail: oguzhan.das@deu.edu.tr

* Corresponding author

https://doi.org/10.1590/1679-78256671

\begin{abstract}
This study presents a free vibration analysis of three different curved laminated composite plates having endto-end decomposition under fixed opposing straight edges. The curved structures have been mathematically modeled by employing the Classical Laminated Plate Theory and the Finite Element Method to perform the location-based effect of decomposition. Besides, the relation among the decomposition, curvature characteristics, and stacking sequences is examined in terms of natural frequencies. For this purpose, the endto-end decompositions are defined in two different levels and re-positioned along the curved edge for each vibration analysis. The frequency ratio is obtained by proportioning the natural frequency of the damaged structure to that of the healthy structure and considered to interpret the effects of the decomposition location. Cross-ply and angle-ply structures having elliptic, circular, and parabolic curvatures are considered. It is concluded that the effect of the end-to-end decomposition on the natural frequencies varies to its level, the stacking order, and the bending regions of the relevant mode shape.
\end{abstract}

\section{Keywords}

free vibration, curved plate, decomposition, laminated composite, finite element analysis

\section{Graphical Abstract}

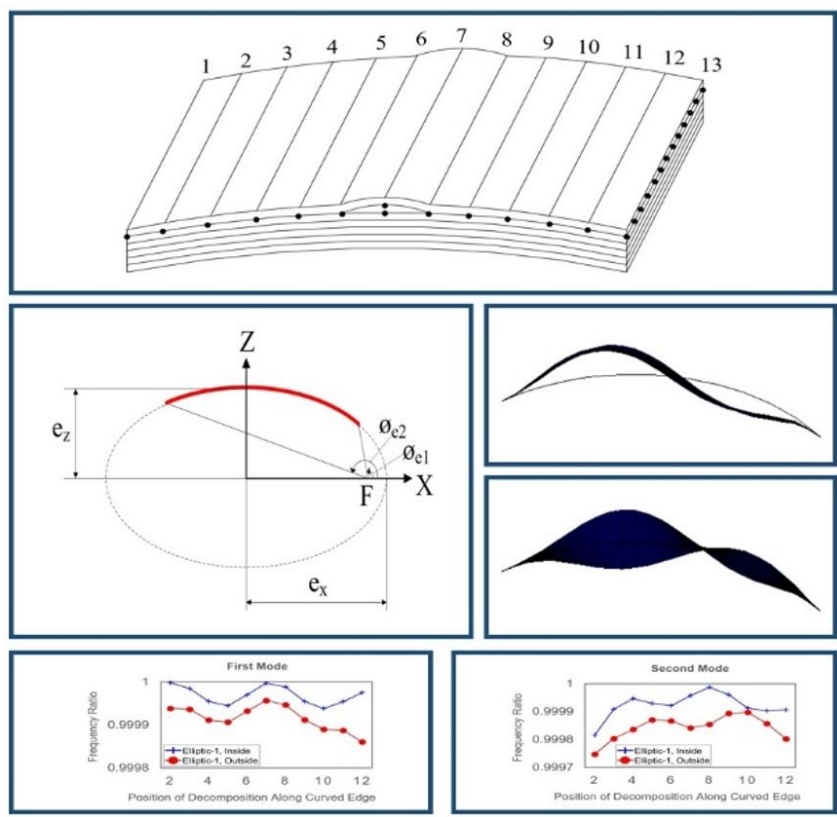

Finite element discretization with decomposition

Elliptical curved model and first two mode shapes for the elliptical curved structure (P1)

Effect of decomposition on free vibration 


\section{INTRODUCTION}

The laminated composite curved plates with different curvature geometries are essential structural components that are extensively used in aerospace, automobile, civil, and other various complex engineering applications. Besides, laminated composites are elegant materials, which used in these fields due to their low weight, high strength, durability against corrosion, and manufacturing flexibility (Dey and Karmakar, 2012). Depending on the production processes and usage areas, any error may occur in the composite plates. These errors directly affect the structure's dynamic characteristics. For this reason, it is of great importance to model the composite plates and the possible damages that they may contain by employing correct modeling techniques before production in order to predict the behavior of curved laminated composite plates under various dynamic loads. On the other hand, different behavioral characteristics of structures due to damage pave the way for reverse engineering processes. This phenomenon can be used for many purposes, especially for damage detection.

There are various studies including healthy and delaminated curved laminated composite structures in the literature. Hirwani et al. (2016) performed the free vibration analysis of delaminated curved panels having cylindrical, spherical, elliptical, and hyperboloid geometries. They measured the effects of the location, size, and severity of the damage within the first six modes of structure. For this purpose, they considered five different delamination locations, four different delamination sizes, and four delamination interfaces. Arumugam and Rajamohan (2017) examined the free and forced vibration characteristics of the rotating thickness-tapered composite plates with delaminations. They employed Classical Plate Theory with Finite Element Method to model the plate structure and perform the free and forced vibration analyses. They measured the effects of the rotational speed, tapered models, size, and position of the delamination on the vibration characteristics of the composite plates. Sahoo et al. (2016) investigated the effects of the delamination on the bending and vibration responses of the laminated composite plate structures using higher-order shear deformation theories. They examined four different delamination locations, sizes, and boundary conditions. Besides, they also measured the effects of the aspect ratio and modular ratio on the same static and dynamic properties. Venkate Gowda et al. (2017) examined the effects of the delamination size and location on the natural frequency of composite beam structures using Finite Element Analysis. They considered two different boundary conditions, three delamination layups, and sizes. Fazilati (2017) investigated the dynamic behavior of variable stiffness composite laminated plate with curvilinear fiber orientation subjected to in-plane end-loads. Wang et al. (2017) considered an arcshaped crack lying along with the interface of a through-thickness circular elastic inhomogeneity in an isotropic laminated thin plate. Tiwari et al. (2019) performed free vibration analysis of composite beams and plates with delaminations by using $3 \mathrm{~d}$ degenerated element method. They considered four delamination interfaces and sizes to measure the delamination effects on the natural frequencies of composite beams and plates having single or multiple delaminations. Kamaloo et al. (2019) performed the non-linear free vibration analysis to measure the effect of the delamination on the vibration response of the composite conical shell having delamination. Besides, they also measured the non-linearity of the number of layers of the conical shells with delamination located at the middle interface of the corresponding structure. He et al. (2019) examined the surface contact effect on the modal damping ratio of the delaminated composite plates. They considered the Finite Element Method to virtually simulate the vibration responses of the composite structures. Juhász and Szekrényes (2020) proposed an analytical model of doubly curved shells to perform buckling and vibration analysis of the delaminated composite spherical shells. For this purpose, they employed the System of Exact Kinematic Conditions and the improved version of the Sanders Shell Theory. They considered various lamination sequences and boundary conditions to validate and show the effectiveness of the proposed method. Kamaloo et al. (2020) performed a nonlinear free vibration analysis to determine the effects of the delamination on the oscillatory motion of the circular cylindrical shells with delamination. For such a purpose, they measured the shell middle surface radius, the delamination length, number of layers, and orthotropy. An et al. (2021) presented a reliability-based design framework for optimal design of composite stacking sequence by considering both delamination and material property uncertainties.

In this study, the relation between the decomposition, the stacking order, and the curvature geometry in terms of the natural frequencies is investigated. For this purpose, elliptic, parabolic, and circular curved plates having cross-ply and angle-ply stacking sequences are considered. In order to validate the accuracy of the healthy curved model, a convergence analysis is performed by using ANSYS. The decomposition is modeled between two opposing end-to-end curved free edges. To correctly interpret the effect of decomposition on free vibration characteristics of the curved structures, the damage is placed to all rows at the free edges in two different levels for all curved structures. The first five natural frequencies and their mode shapes are obtained for all curved decomposed laminated composite plates. Frequency ratios are used to interpret the location-based effect of decomposition, which are obtained by proportioning the free vibration responses of the damaged plates to the healthy plates. 


\section{MATHEMATICAL MODEL}

\subsection{Finite element model}

In the case of the shell thickness is larger than around $10 \%$ of the plate length, shear deformation has a countable effect. As a result of this phenomenon, the Classical Laminated Plate Theory (CLPT) works for very thin and moderately thin plates, while higher-order plate theories satisfy for thick plates (Groh and Weaver, 2015; Donnell, 1976). The CLPT is used in this study since thin plates are included in the scope of the study. Accordingly, it should be noted that the effect of the shear deformation on the structure is negligible. The Finite Element Analysis is performed by employing a fournode quadrilateral element. Figure 1 shows the illustration of the employed laminated finite element.

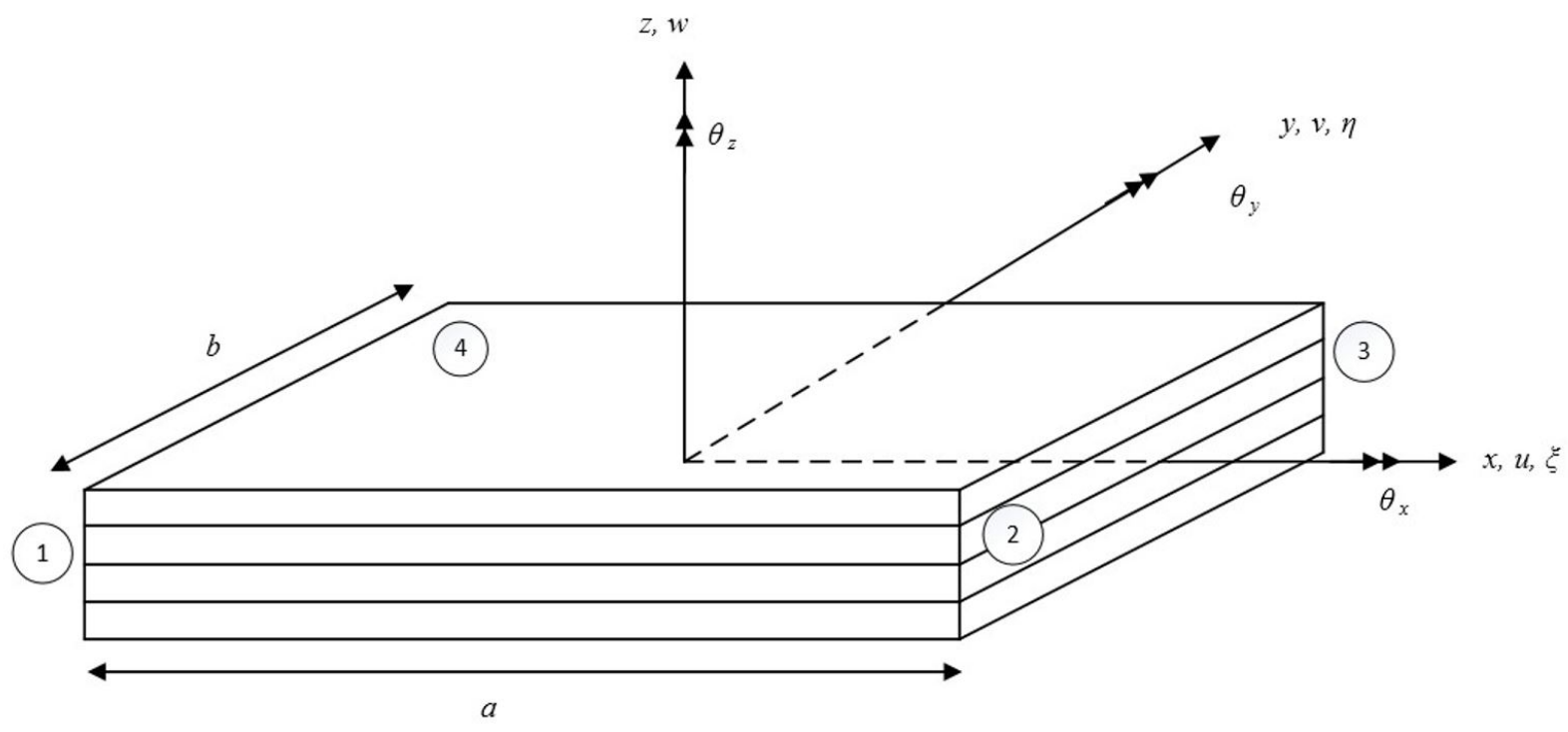

Figure 1 Degrees of freedom of the laminated finite element.

Normals to the middle surface of the undeformed plate remain straight and normal to the middle surface during deformation and are inextensible for in-plane movement (Petyt, 2010). Thus, the components of in-plane displacements $u$ and $v$ expressed as

$v=q_{5}+q_{6} \mathrm{x}+q_{7} \mathrm{y}+q_{8} x y$

$$
u=q_{1}+q_{2} \mathrm{x}+q_{3} \mathrm{y}+q_{4} x y
$$

where $q_{1}-q_{8}$ are the coefficients of the in-plane displacements. The displacement functions are required and that is

$v=\sum_{j=1}^{4} N_{1 j} v_{j}$

$$
u=\sum_{j=1}^{4} N_{1 j} u_{j}
$$

where $N_{1 j}$ represents the shape functions. The shape functions for the in-plane displacements in terms of the natural coordinates $\left(\xi, \eta_{j}\right)$ of node $j$ can be expressed as follows (Petyt, 2010; Chandrupatla and Belegundu, 2002)

$N_{1 j}=\frac{1}{4}\left(1+\xi_{j} \xi\right)\left(1+\eta_{j} \eta\right)$

The displacement function for bending displacement is

$w=q_{9}+q_{10} \xi+q_{11} \eta+q_{12} \xi^{2}+q_{13} \xi \eta+q_{14} \eta^{2}+q_{15} \xi^{3}+q_{16} \xi^{2} \eta+q_{17} \xi \eta^{2}+q_{18} \eta^{3}+q_{19} \xi^{3} \eta+q_{20} \xi \eta^{3}$ 
where $q_{9}-q_{20}$ are the coefficients of the bending displacement. The displacement function is required and that is

$w=\left[N_{21}(\xi, \eta) N_{22}(\xi, \eta) N_{23}(\xi, \eta) N_{24}(\xi, \eta)\right]\{w\}_{e}=\left[N_{2}(\xi, \eta)\right]\{w\}_{e}$

where $\{w\}_{e}$ element displacements. Shape function $\left(N_{2 j}\right)$ of out-of-plane movement for the ${ }^{\text {th }}$ node is given as in equation (6) (Petyt, 2010)

$N_{2 j}^{T}(\xi, \eta)=\left[\begin{array}{c}\frac{1}{8}\left(1+\xi_{j} \xi\right)\left(1+\eta_{j} \eta\right)\left(2+\xi_{j} \xi+\eta_{j} \eta-\xi^{2}-\eta^{2}\right) \\ \frac{b}{2}\left(1+\xi_{j} \xi\right)\left(\eta_{j}+\eta\right)\left(\eta^{2}-1\right) \\ -\frac{a}{2}\left(\xi_{j}+\xi\right)\left(\xi^{2}-1\right)\left(1+\eta_{j} \eta\right)\end{array}\right]$

where $a$ and $b$ denote the dimensions of the plate element. Both in-plane and out-of-plane displacements must be taken into account as they are coupled for some stacking orders (Petyt, 2010; Bathe, 2014). The matrix form of the strain equation can be expressed as follows

$\{\varepsilon\}=\left\{\begin{array}{c}\varepsilon_{x} \\ \varepsilon_{y} \\ \Upsilon_{x y}\end{array}\right\}=\left[\begin{array}{c}\frac{\partial u_{0}}{\partial x} \\ \frac{\partial v_{0}}{\partial y} \\ \frac{\partial u_{0}}{\partial y}+\frac{\partial v_{0}}{\partial x}\end{array}\right]-z\left[\begin{array}{c}\frac{\partial^{2} w_{0}}{\partial x^{2}} \\ \frac{\partial^{2} w_{0}}{\partial y^{2}} \\ 2 \frac{\partial^{2} w_{0}}{\partial x \partial y}\end{array}\right]$

where $u_{0}, v_{0}$, and $w_{0}$ are the displacement components at a point $(x, y)$ in the middle surface. Equation (7) can be expressed as

$\{\varepsilon\}=\left\{\varepsilon_{0}\right\}-z\left\{\chi_{0}\right\}$

Equation (9) gives the strain energy of the thin plate as

$U=\frac{1}{2} \int_{V}\{\varepsilon\}^{T}\{\sigma\} d V$

where $\{\sigma\}$ is the stress vector, and $\{\varepsilon\}$ is the strain vector. The stress-strain relation can be stated as in equation (10) for a thin plate

$\{\sigma\}=[D]\{\varepsilon\}$

where $[D]$ is a material matrix. The strain energy is formed as

$U=\frac{1}{2} h \int_{A}\{\varepsilon\}^{T}[D]\{\varepsilon\} d A$

where $h$ is the plate thickness. Equation (11) can be rewritten for a laminated plate as

$U=\frac{1}{2} \int_{A} \sum_{k=1}^{N L} \int_{z_{k-1}}^{z_{k}}\{\varepsilon\}^{T}\left[D^{k}\right]\{\varepsilon\} d z d A$

Here, $N L$ denotes the number of laminae, $z_{k}$ and $z_{k-1}$ are the top and bottom coordinates of the $k^{\text {th }}$ lamina. [ $\left.D^{k}\right]$ is given by equations (13)-(15).

$\left[D^{*}\right]^{k}=\left[\begin{array}{ccc}\frac{E_{x}}{1-v_{x y} v_{y x}} & \frac{E_{x} v_{x y}}{1-v_{x y} v_{y x}} & 0 \\ & \frac{E_{y}}{1-v_{x y} v_{y x}} & 0 \\ S y m & & G_{x y}\end{array}\right]$ 
$E_{x}$ is the modulus of elasticity in the $x$-direction, $E_{y}$ is the modulus of elasticity in the $y$-direction, $v_{x y}$ and $v_{y x}$ are the Poisson's ratios that correspond to a contraction in direction of the second subscript when an extension is applied in direction of the first subscript, $G_{x y}$ is the shear modulus. In general, the material axes that forming each layer of the plate would be inclined at an angle $B$ to the geometric axes. Considering the relationship between material and geometric axes, the matrix of material constants is formed as follows

$\left[D^{k}\right]=\left[R t^{*}\right]^{T}\left[D^{*}\right]^{k}\left[R t^{*}\right]$

where the transformation matrix $\left[R t^{*}\right]$ is given by

$\left[R t^{*}\right]=\left[\begin{array}{ccc}\cos ^{2} \beta & \sin ^{2} \beta & 0.5 \sin 2 \beta \\ \sin ^{2} \beta & \cos ^{2} \beta & -0.5 \sin 2 \beta \\ -\sin 2 \beta & \sin 2 \beta & \cos 2 \beta\end{array}\right]$

Here, $b$ is the fiber angle with respect to the $x$-axis. Substituting equation (8) into equation (12) and integrating with respect to $z$ gives

$U=\frac{1}{2} \int_{A}\left(\left\{\varepsilon_{0}\right\}^{T}[A]\left\{\varepsilon_{0}\right\}-\left\{\varepsilon_{0}\right\}^{T}[B]\left\{\chi_{0}\right\}-\left\{\chi_{0}\right\}^{T}[B]\left\{\varepsilon_{0}\right\}+\left\{\chi_{0}\right\}^{T}[C]\left\{\chi_{0}\right\}\right) d A$

where

$[C]=\frac{1}{3} \sum_{k=1}^{N L}\left[D^{k}\right]\left(z_{k}^{3}-z_{k-1}^{3}\right)$

$$
\begin{aligned}
& {[A]=\sum_{k=1}^{N L}\left[D^{k}\right]\left(z_{k}-z_{k-1}\right)} \\
& {[B]=\frac{1}{2} \sum_{k=1}^{N L}\left[D^{k}\right]\left(z_{k}^{2}-z_{k-1}^{2}\right)}
\end{aligned}
$$

Equations (2), (5), and (7) are substituted in equation (16), and rearranged to obtain equation (18):

$U=\frac{1}{2}\{q\}^{T}[k]\{q\}$

where $[k]$ represents the element stiffness matrix in dimension (20x20). The kinetic energy of the plate is given by equation (19) with the average density $\rho$ and the thickness of the laminate is $h$.

$T=\frac{1}{2} \int_{A} \rho h\left(\dot{u}^{2}+\dot{v}^{2}+\dot{w}^{2}\right) d A$

Equation (19) is rearranged using equations (2) and (5)

$T=\frac{1}{2}\{\dot{q}\}^{T}[m]\{\dot{q}\}$

here, $[\mathrm{m}]$ represents the $(20 \times 20)$ element mass matrix. To satisfy the degrees of freedom of the curved structures, the drilling effect $\left(\vartheta_{z}\right)$ has to be considered. Hence the element stiffness and mass matrices are expanded to (24x24) from (20x20). The corresponding diagonal values of $\left[\vartheta_{z}\right]_{4 \times 4}$ have taken absolute value that $1 / 1000$ of the minimum value in (20x20) stiffness and mass matrices, while other values of $\left[\vartheta_{z}\right]_{4 \times 4}$ are considered to be zero (Guha Niyogi et al. 1999, and Gonenli and Das, 2021). The generalized displacement vector of the jth node for local reference coordinates is expressed as

$\left\{q_{j}\right\}=\left[w_{j} \theta_{x_{j}} \theta_{y_{j}} u_{j} v_{j} \theta_{z_{j}}\right]$ 
Figure 2 shows the rotation of the coordinate system with respect to the $y$-axis to model the curved structures where $\theta$ represents the rotation angle.

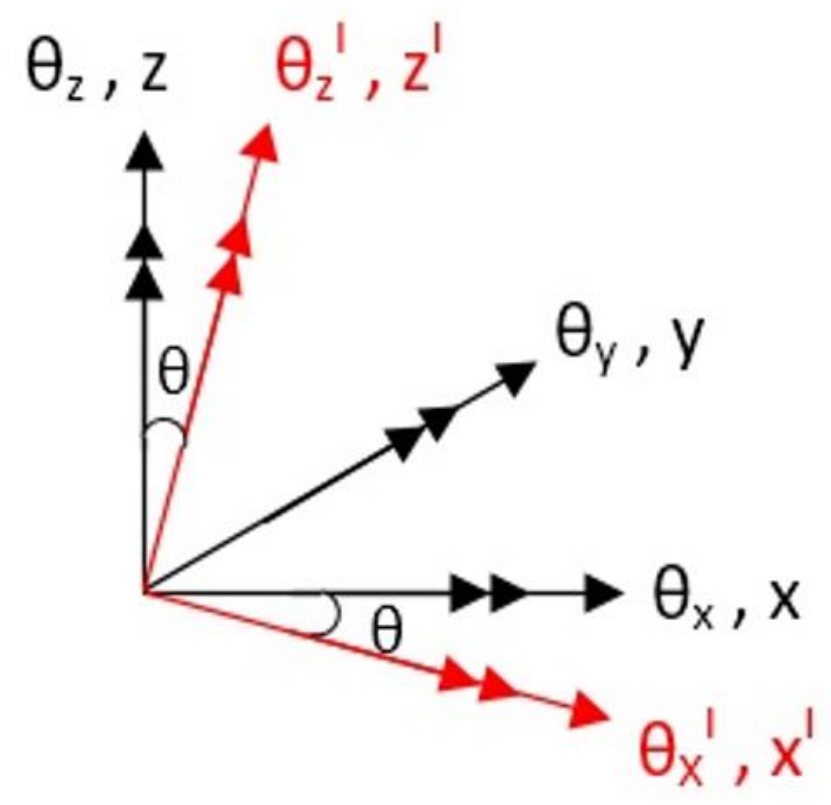

Figure 2 The rotation of the coordinate system around the $y$-axis.

The transformation matrix is given in equation (22).

$\operatorname{Tr}=\left[\begin{array}{cccccc}\cos (\theta) & 0 & 0 & -\sin (\theta) & 0 & 0 \\ 0 & \cos (\theta) & 0 & 0 & 0 & \sin (\theta) \\ 0 & 0 & 1 & 0 & 0 & 0 \\ \sin (\theta) & 0 & 0 & \cos (\theta) & 0 & 0 \\ 0 & 0 & 0 & 0 & 1 & 0 \\ 0 & -\sin (\theta) & 0 & 0 & 0 & \cos (\theta)\end{array}\right]$

The dynamic response of a plate for the entire system can be formulated via Lagrange's equation of motion as (Petyt, 2010)

$[M]\{\ddot{\mathrm{q}}\}+[K]\{\mathrm{q}\}=0$

where $[K],[M]$, and $\{q\}$ represent the global stiffness matrix, global mass matrix, and global displacement vector, respectively. The natural frequencies can be calculated through equation (24) as an eigenvalue problem, where $\omega$ represents the natural frequency as

$\left([K]-\omega^{2}[M]\right)\{q\}=0$

\subsection{Decomposition model}

In laminated structures, there may be a segregation error along an axis between laminates, called decomposition. To accurately interpret the decomposition effect on the dynamic characteristics of the structure, it is required that the damage and the decomposition should be correctly modelled and represented. For this purpose, new nodes can be introduced on the plate along the axis where separation takes place. The illustration of this modeling is presented in Figure 3 in which Figure 3 (a) indicates the healthy laminated plate model, Figure 3 (b) shows the laminated plate model where the decomposition is close to the neutral axis of the plate, and Figure 3 (c) shows the laminated plate model that includes the decomposition in the outermost layer. 


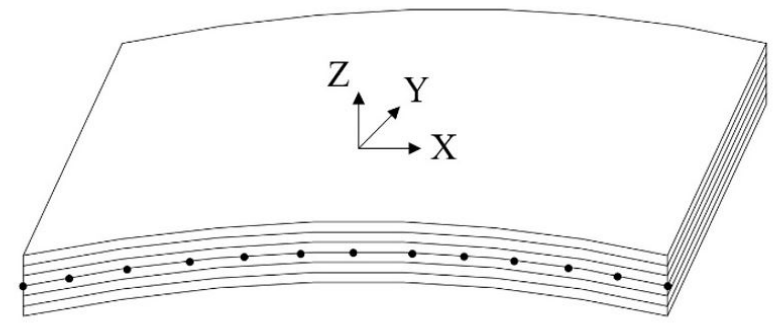

(a)

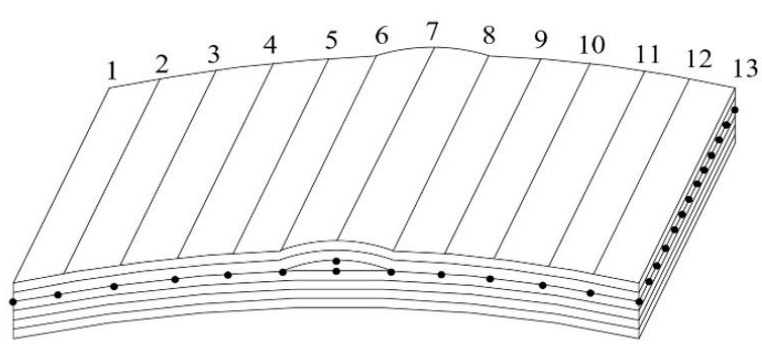

(b)

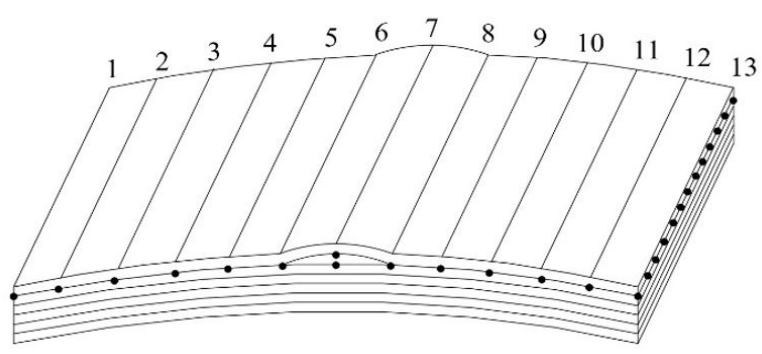

(c)

Figure 3: Laminated composite plate models: (a) Healty model, (b) Laminated plate model with decomposition in inside position, (c) Laminated plate model with decomposition in outside position.

To investigate the effect of the decomposition on the free vibration characteristics of the structure, the decomposition is modeled along the curved edges and repositioned through the plate for both decomposition levels given in Figures 3 (b) and 3 (c) to also measure its location-wise effects.

\subsection{Curvature geometries}

In engineering structures, various curved geometries are generally preferred to achieve high rigidity for specific engineering purposes and for aesthetic reasons. In this study, elliptical, circular, and parabolic types of curves are considered. The elliptical, circular, and parabolic curves with the relevant geometrical parameters are given in Figure 4 (Qin et al., 2020).

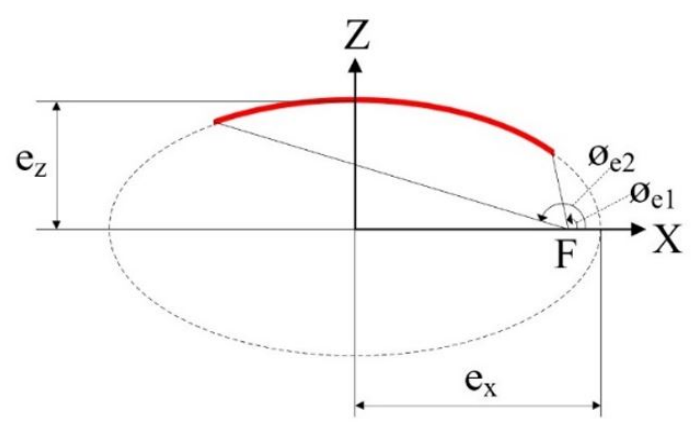

(a)

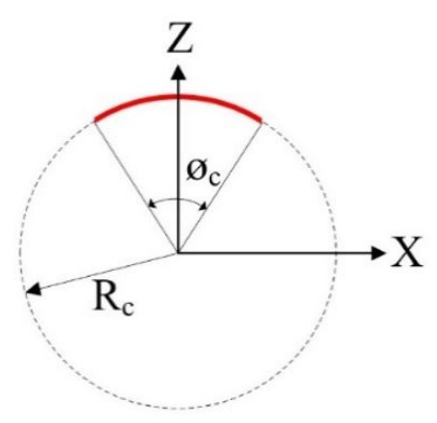

(b)

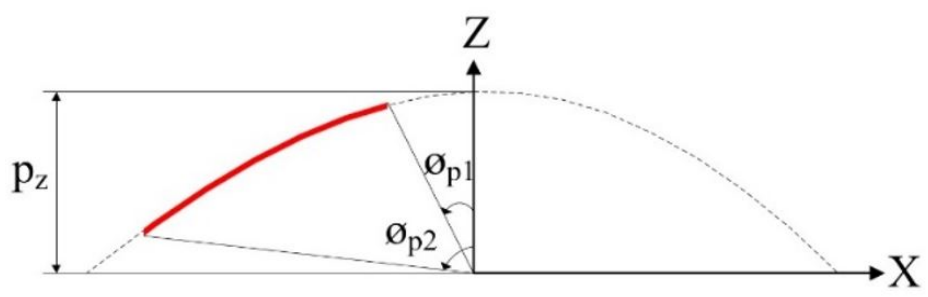

(c)

Figure 4: Different types of curved plate structures: (a) Elliptic curved plate, (b) Circular curved plate, (c) Parabolic curved plate. 
The symbols given in Figure 4 are the drawing parameters of the curved geometries. In Figure 4 (a), $\varnothing_{e 1}$ and $\emptyset_{e 2}$ represent the starting and ending angles of the ellipse, $e_{x}$ and $e_{z}$ represent the width and height of the ellipse, and $F$ represents the ellipse's focus point. The $\emptyset_{c}$ in Figure 4 (b) represents the angle of the circular segment, while $R_{c}$ represents the radius of the circle. While $\varnothing_{p 1}$ and $\emptyset_{p 2}$ in Figure 4 (c) represent the starting and ending angles of the parabola, $p_{z}$ gives the height of the parabola.

\section{RESULTS}

The six-layer laminated composite plates with three different curvatures and two different stacking orders are examined in this study. The geometric features, material properties, and numerical information of the laminated composite plates are given in Table 1.

Table 1 Material properties and the geometry of the curved plates (Gay et al., 2003; Ozturk, 2015)

\begin{tabular}{ccc}
\hline Symbol & Name & Quantity, Order \\
\hline$E_{x}$ & Elasticity modulus in the x-direction & $45 \mathrm{GPa}$ \\
$E_{Y}$ & Elasticity modulus in the y-direction & $12 \mathrm{GPa}$ \\
$\rho$ & Mass density & $2080 \mathrm{~kg} / \mathrm{m}^{3}$ \\
$v_{x y}$ & Major Poisson's ratio & 0.30 \\
$v_{y x}$ & Minor Poisson's ratio & $\left(E_{y} / E_{x}\right) v_{x y}$ \\
$e_{x}$ & Width of the ellipse & $0.3 \mathrm{~m}$ \\
$e_{z}$ & Height of the ellipse & $0.1 \mathrm{~m}$ \\
$R_{c}$ & Radius of the circle & $0.6 \mathrm{~m}$ \\
$p_{z}$ & Height of the parabola & $0.6 \mathrm{~m}$ \\
$\emptyset_{e 1}-\emptyset_{e 2}$ & Angles of the ellipse & $120^{\circ}-170^{\circ}$ \\
$\emptyset_{c}$ & Segment angle of the circle & $45^{\circ}$ \\
$\emptyset_{p 1}-\emptyset_{p 2}$ & Angles of the parabola & $20^{\circ}-60^{\circ}$ \\
$h$ & Thickness of the plate element & $0.006 \mathrm{~m}$ \\
$P_{1}$ & Mesh density & $12 \times 12$ \\
$P_{2}$ & Stacking order - 1 & {$\left[45^{\circ} /-45^{\circ} / 45^{\circ}\right]_{2}$}
\end{tabular}

The non-dimensional natural frequency parameter $(\lambda)$ is given in Equation (25), where $A$ denotes the cross-sectional area of the structure and $/$ denotes the moment of inertia.

$\lambda=\omega \sqrt{\frac{\rho A a^{4}}{E_{y} I}}$

Free vibration analyses of healthy and damaged laminated composite curved plates are performed on the plate models given in Figure 3, by fixing opposing straight sides. All analyses are performed by employing a computer code written in MATLAB for computational efficiency since conducting consecutive numerous analyses via Computer-Aided Design software significantly increases the computational time. To validate the considered numerical model, a convergence analysis is performed with ANSYS. In this context, a number of 12x12 SHELL181 elements are considered. Table 2 presents the first five natural frequencies for two different lamination orientations of three different curved plates in terms of non-dimensional natural frequency parameters. It is seen that the finite element model accurately represents the laminated composite healthy plate in different types of curvatures and stacking orders. 
Table 2 Convergence analysis of the curved plates for two different stacking orders

\begin{tabular}{|c|c|c|c|c|c|c|c|c|c|}
\hline & \multicolumn{3}{|c|}{ Elliptic Curved Plate - P1 } & \multicolumn{3}{|c|}{ Circular Curved Plate - P1 } & \multicolumn{3}{|c|}{ Parabolic Curved Plate - P1 } \\
\hline & Present & ANSYS & Diff. & Present & ANSYS & Diff. & Present & ANSYS & Diff. \\
\hline$\lambda_{1}$ & 13.52 & 13.24 & $2.1 \%$ & 13.77 & 13.39 & $2.8 \%$ & 13.70 & 13.54 & $1.1 \%$ \\
\hline$\lambda_{2}$ & 19.33 & 18.96 & $2.0 \%$ & 23.13 & 22.44 & $3.1 \%$ & 17.31 & 17.08 & $1.4 \%$ \\
\hline$\lambda_{3}$ & 19.68 & 19.43 & $1.3 \%$ & 24.10 & 23.61 & $2.1 \%$ & 17.85 & 17.71 & $0.8 \%$ \\
\hline$\lambda_{4}$ & 21.54 & 21.09 & $2.1 \%$ & 25.75 & 25.03 & $2.9 \%$ & 18.94 & 18.69 & $1.3 \%$ \\
\hline \multirow[t]{3}{*}{$\lambda_{5}$} & 26.40 & 25.86 & $2.1 \%$ & 35.80 & 34.62 & $3.4 \%$ & 22.35 & 22.00 & $1.6 \%$ \\
\hline & \multicolumn{3}{|c|}{ Elliptic Curved Plate - P2 } & \multicolumn{3}{|c|}{ Circular Curved Plate - P2 } & \multicolumn{3}{|c|}{ Parabolic Curved Plate - P2 } \\
\hline & Present & ANSYS & Diff. & Present & ANSYS & Diff. & Present & ANSYS & Diff. \\
\hline$\lambda_{1}$ & 17.21 & 16.85 & $2.1 \%$ & 17.49 & 17.02 & $2.8 \%$ & 17.45 & 17.26 & $1.1 \%$ \\
\hline$\lambda_{2}$ & 21.24 & 20.74 & $2.4 \%$ & 23.80 & 23.02 & $3.4 \%$ & 19.68 & 19.41 & $1.4 \%$ \\
\hline$\lambda_{3}$ & 25.10 & 24.78 & $1.3 \%$ & 30.56 & 29.95 & $2.1 \%$ & 22.92 & 22.77 & $0.7 \%$ \\
\hline$\lambda_{4}$ & 25.93 & 25.47 & $1.8 \%$ & 31.53 & 30.66 & $2.8 \%$ & 23.39 & 23.18 & $0.9 \%$ \\
\hline$\lambda_{5}$ & 28.51 & 27.92 & $2.1 \%$ & 36.01 & 34.81 & $3.5 \%$ & 24.81 & 24.48 & $1.3 \%$ \\
\hline
\end{tabular}

The first five mode shapes of the elliptic curved model are the same for $\left[45^{\circ} /-45^{\circ} / 45^{0}\right]_{2}(\mathrm{P} 1)$ and $\left[0^{0} / 90^{\circ} / 0^{\circ}\right]_{2}(\mathrm{P} 2)$. The first five mode shapes of the elliptic curve model are given in Figure 5.

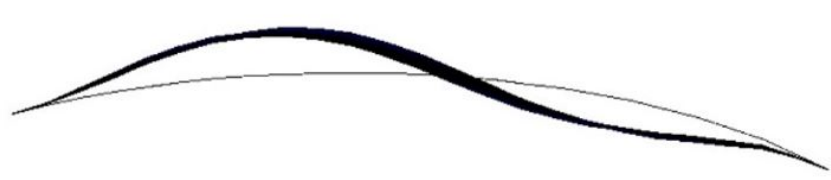

(a)

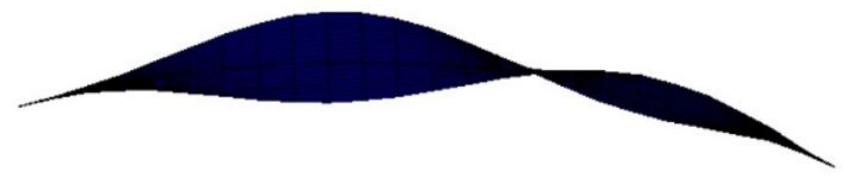

(b)

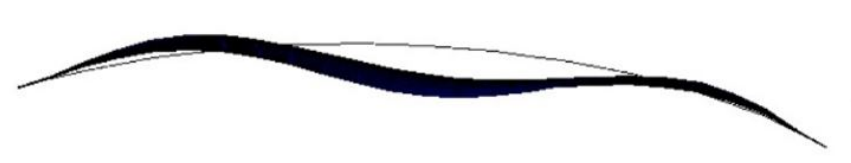

(c)

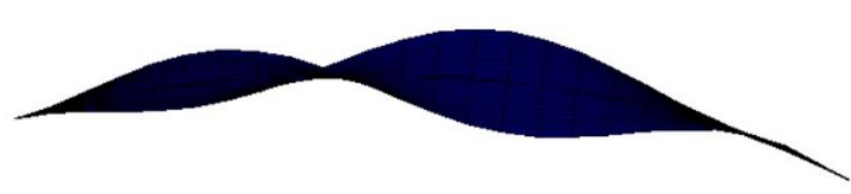

(d)

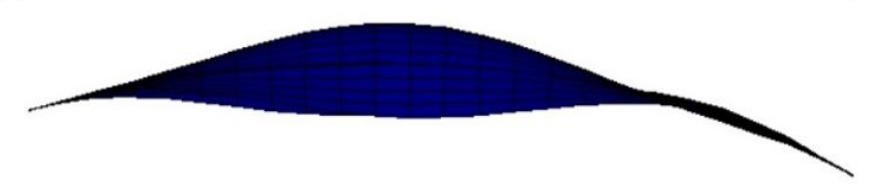

(e)

Figure 5: The first five mode shapes of the elliptic curved plate for all stacking orders.

The first five mode shapes of the circular curve model for the two different stacking orders are given in Figure 6 . It is seen that the mode shapes do not differ no matter which stacking order is considered. 


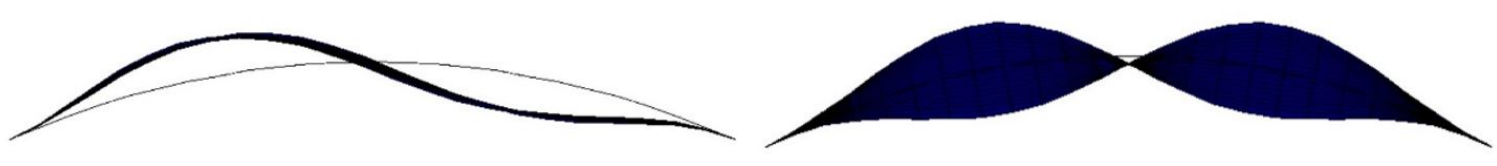

(a)

(b)

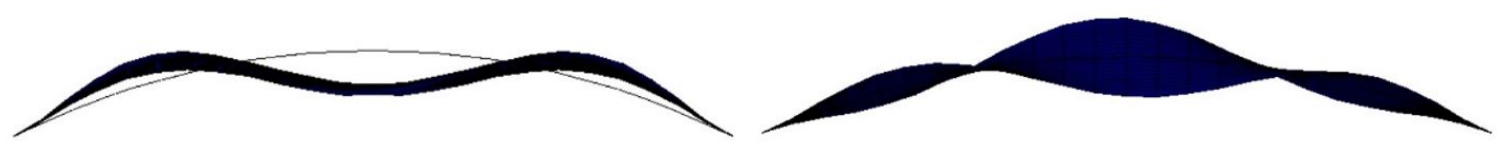

(c)

(d)

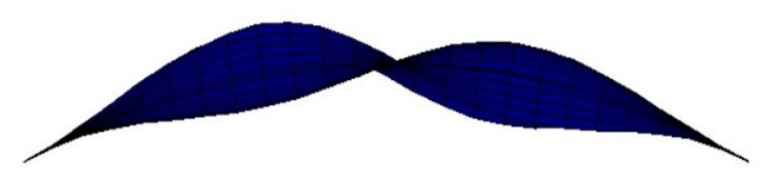

(e)

Figure 6: The first five mode shapes of the circular curved plate for all stacking orders.

The first four mode shapes of the parabolic curved structure, shown in Figures 7 (a) -7 (d) are the same for two different stacking orders. On the other hand, The fifth mode shape of this structure varies for the P1 and P2 stacking sequences as seen in Figures 7 (e) and 7 (f), respectively.

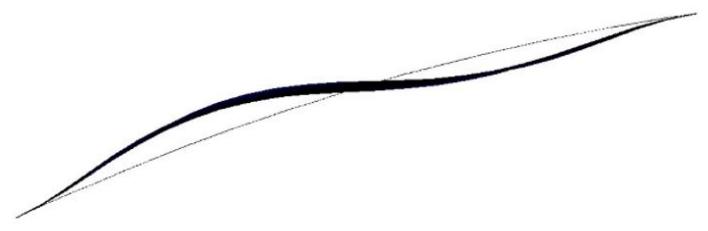

(a)

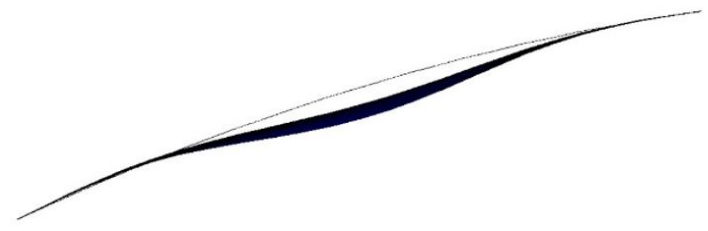

(c)

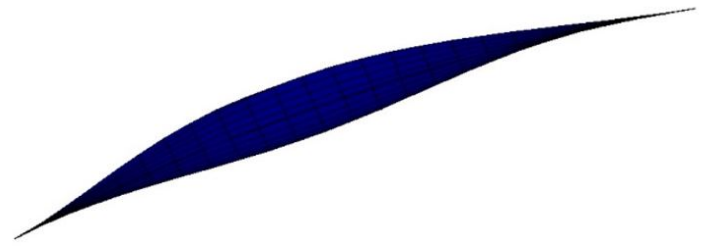

(e)

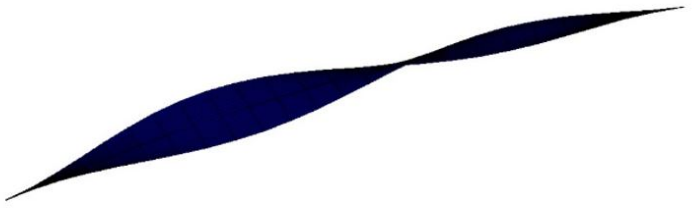

(b)

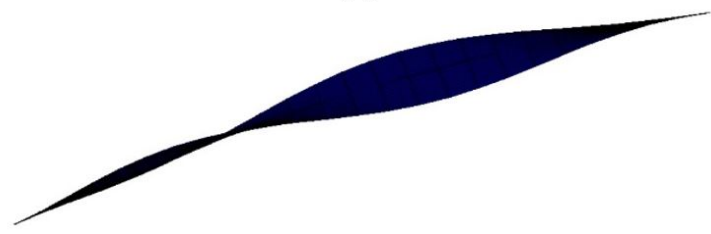

(d)

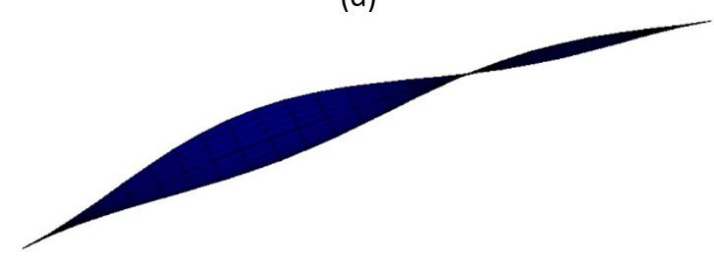

(f)

Figure 7: The first four five mode shapes $(a, b, c, d)$ and the fifth mode shape for P1 (e) and P2 (f) of the parabolic curved plate.

As is mentioned in Section 2.2, the decomposition is repositioned along curved edges except for the fixed edges (rows 1 and 13). The "Frequency Ratio" expression in the figures represents the ratio of the natural frequency value for the damaged model to the healthy model. Figures 8 (a), (c), (e), (g), (i) show the first five natural frequency ratios of the elliptically curved plate for the P1. Figure $8(b),(d),(f),(h),(j)$ show the first five natural frequency ratios of the elliptically curved plate for the P2. The expression "Inside" in the figures represents the decomposition is close to the neutral axis as it is illustrated in Figure 3 (b), and the expression "Outside" represents the decomposition is in the outer lamination as it is shown in Figure 3 (c). 


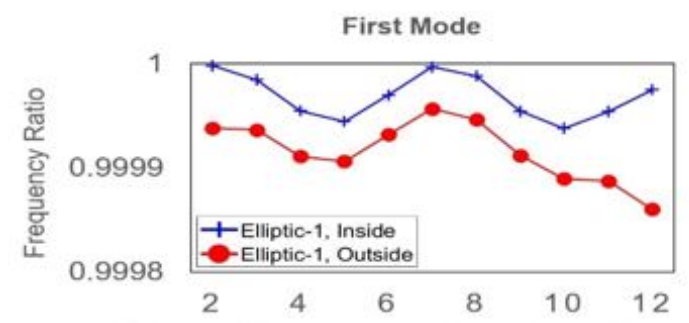

Position of Decomposition Along Curved Edge

(a)

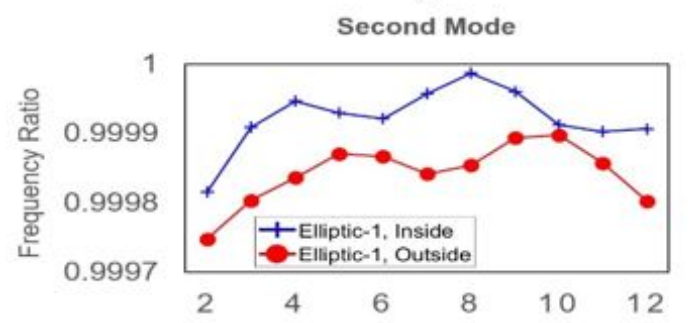

Position of Decomposition Along Curved Edge

(c)

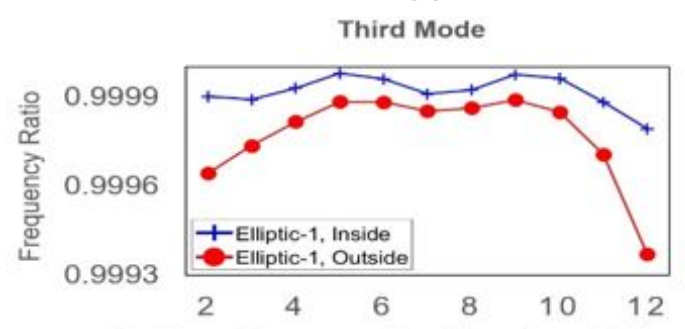

Position of Decomposition Along Curved Edge

(e)

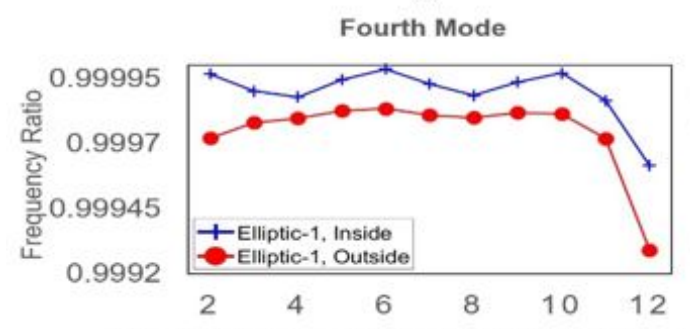

Position of Decomposition Along Curved Edge

(g)

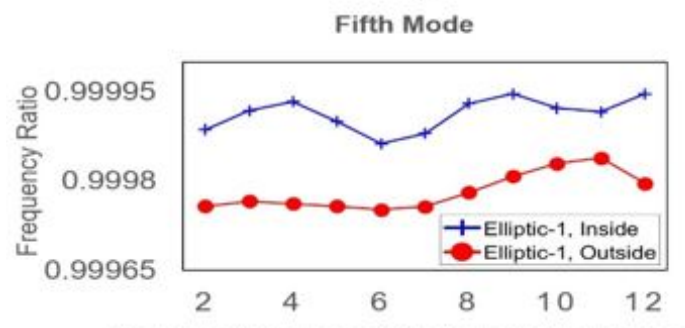

Position of Decomposition Along Curved Edge

(i)

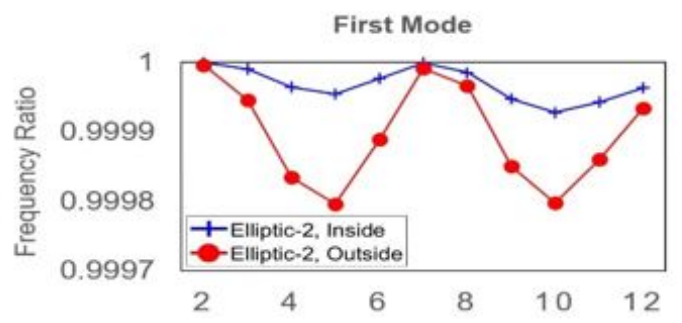

Position of Decomposition Along Curved Edge

(b)

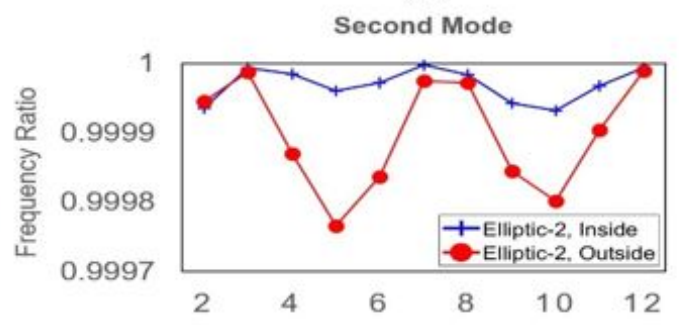

Position of Decomposition Along Curved Edge

(d)

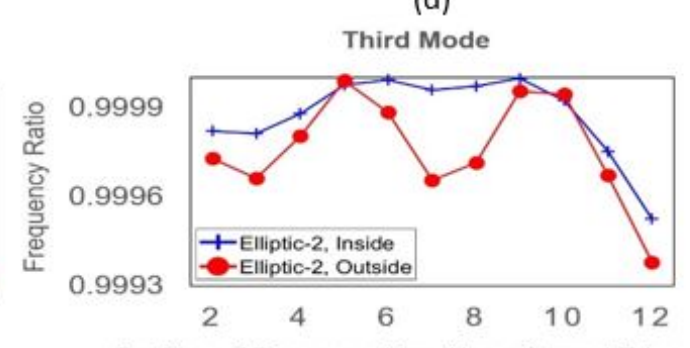

Position of Decomposition Along Curved Edge

(f)

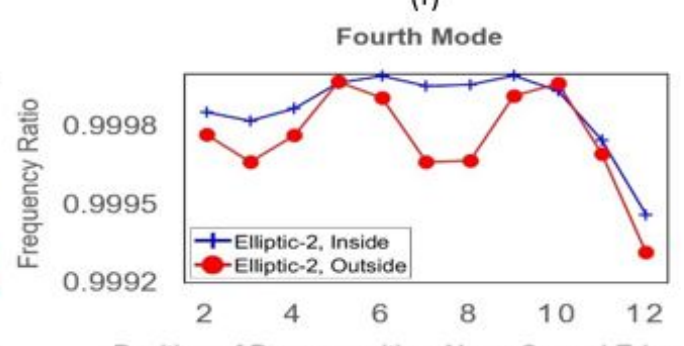

Position of Decomposition Along Curved Edge

(h)

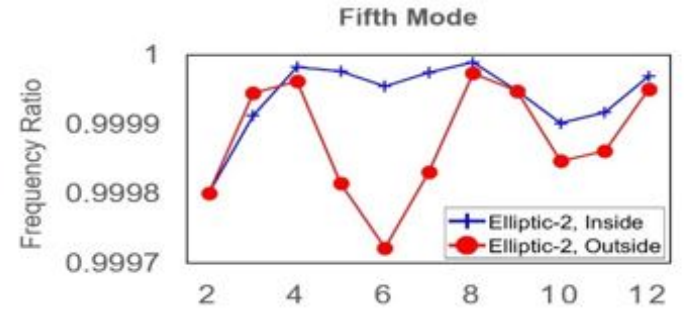

Position of Decomposition Along Curved Edge

(j)

Figure 8: Decomposition effects in elliptic curved plate.

The frequency ratios vary depending on the relevant mode shapes and the layer where the decomposition is located. For instance, the existence of the decomposition in the middle of the plate (i.e., $7^{\text {th }}$ row in Figure 8 (a)) does not make a considerable impact on the first natural frequency of the structure when compared with those of occurred in other regions of the curved plate. This is because there is no bending zone that occurred in the first mode shape of the elliptically curved structure as seen in Figure 5 (a). Especially if the decomposition occurs closer to the neutral axis, this frequency ratio is almost ineffective, that is, it approaches 1 . However, for the first mode shape, a considerable effect of 
the decomposition on the frequency ratio can be observed at the $12^{\text {th }}$ row as seen in Figure 8 (a). The separation of only the outermost $45^{\circ}$ layer of the P1 plate and the separation of both outermost layers, $-45^{\circ}$ and $45^{\circ}$ of the plate constitute opposite effects. A similar situation is not observed for the P2 structure.

The first mode shapes are formed based on bending in elliptically curved plates. For this reason, the modulus of elasticity in the $x$-direction has an active role in the vibration. When both the outermost $45^{\circ}$ and $-45^{\circ}$ layers separate together from the plate, it causes a different response compared to the separation of only the outermost $45^{\circ}$ layer. However, when the outermost $90^{\circ}$ and $0^{\circ}$ layers separate together from the plate, it produces a similar response with that of the condition when only the outermost $0^{\circ}$ layer separates. This is because of the reduction of the elastic modulus in the $x$-direction of the structure due to $90^{\circ}$ fiber angle. Therefore, it can be concluded that the level of the decomposition and the fiber orientation constitutes a coupled effect on the natural frequencies of the elliptic structures.

Figures 9 (a), (c), (e), (g), (i) show the frequency ratios of the circular curved plate for the P1, and Figures 9 (b), (d), (f), (h), (j) show the frequency ratios of the circular curved plate for the P2.

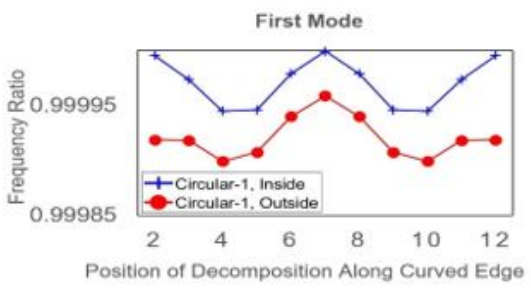

(a)

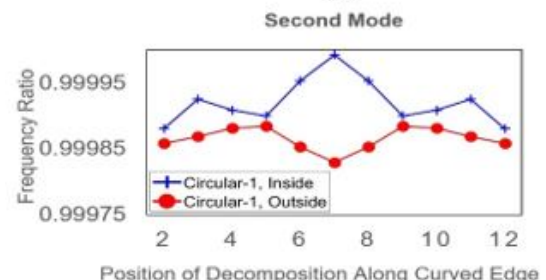

(c)

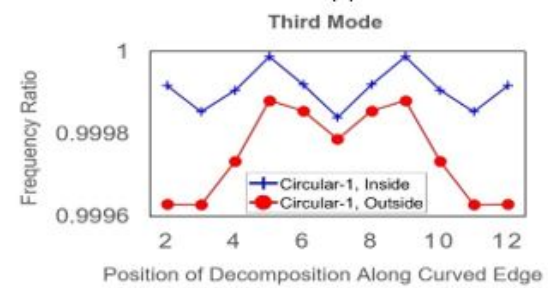

(e)

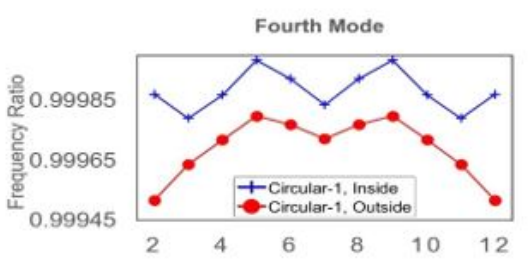

Position of Decomposition Along Curved Edge

(g)

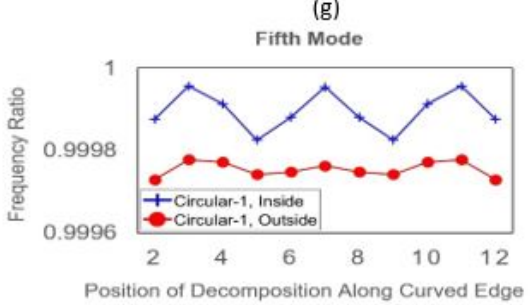

(i)

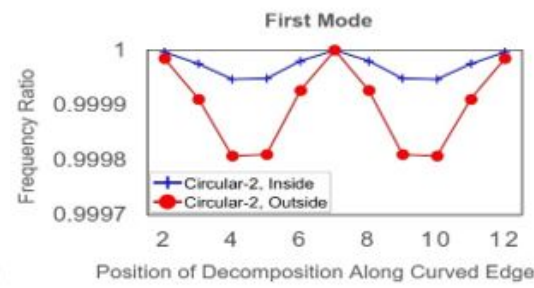

(b)

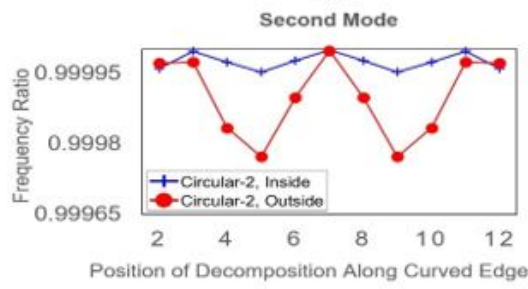

(d)

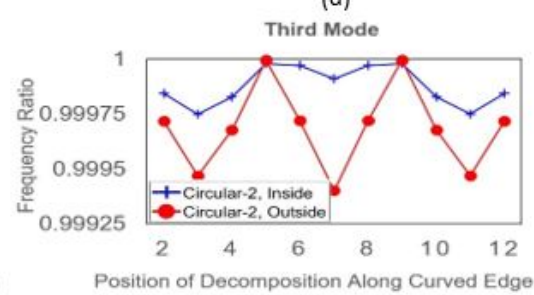

(f)

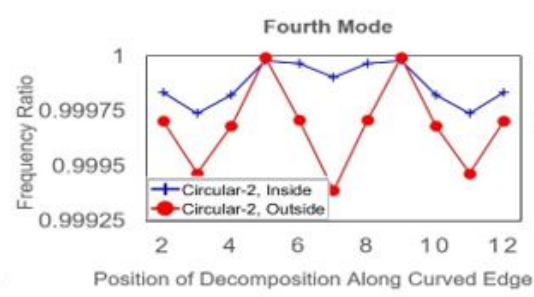

(h)

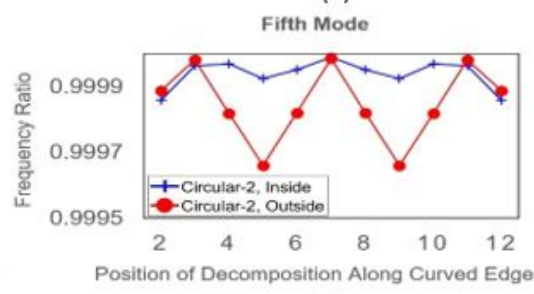

(j)

Figure 9: Decomposition effects in circular curved plate.

According to Figure 9, it can be concluded that the effect of the damage decreases as the decomposition position is closer to the neutral axis in circular curve laminated composite plates for both stacking orders. However, the effect of 
damage on the structure becomes more apparent in the bending regions of the mode shapes of the circular structure. In general, an outside decomposition affects the vibration characteristics of the circular curved structure more than that of an inside decomposition. On the other hand, exceptions may occur where inside and outside decompositions affect the natural frequencies in the opposite way due to the stacking order (e.g., Figure 9 (c) $-7^{\text {th }}$ row).

Figures 10 (a), (c), (e), (g), (i) show the frequency ratios of the parabolic curved plate for the P1, and Figures 10 (b), (d), (f), (h), (j) show the frequency ratios of the parabolic curved plate for the P2, respectively.

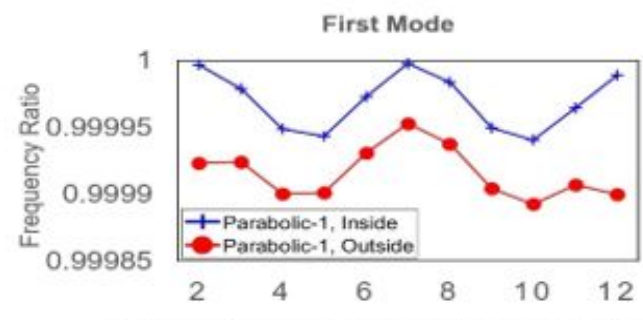

Position of Decomposition Along Curved Edge

(a)

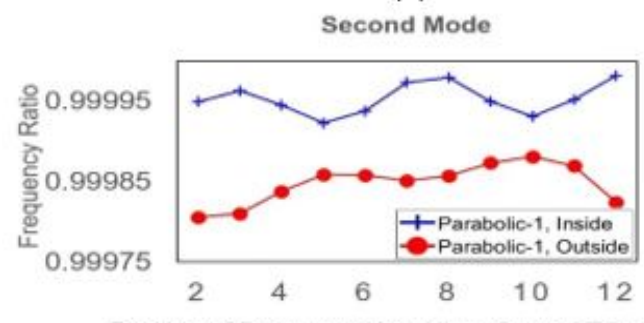

Position of Decomposition Along Curved Edge

(c)

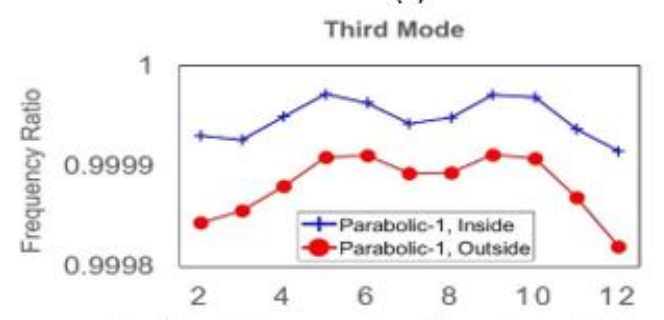

Position of Decomposition Along Curved Edge

(e)

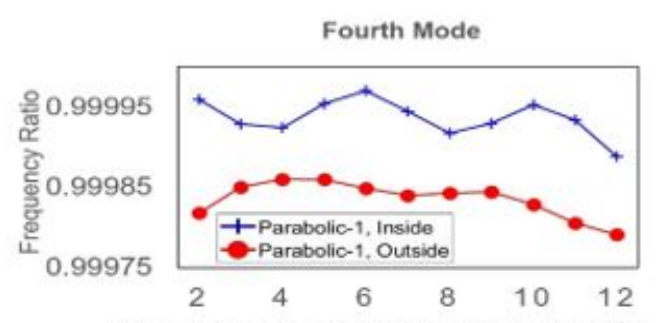

Position of Decomposition Along Curved Edge

(g)

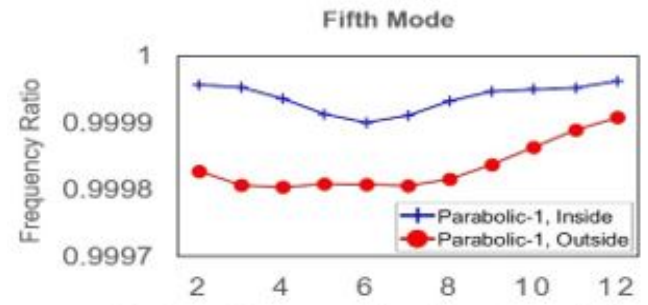

Position of Decomposition Along Curved Edge

(i)

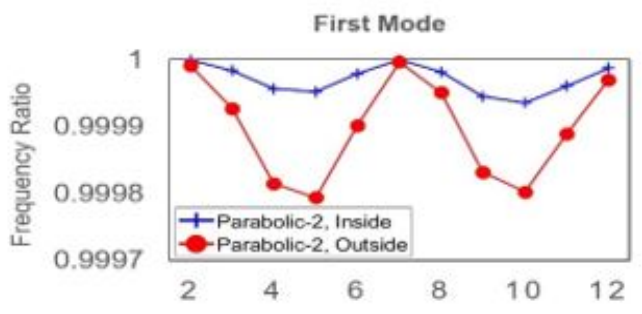

Position of Decomposition Along Curved Edge

(b)

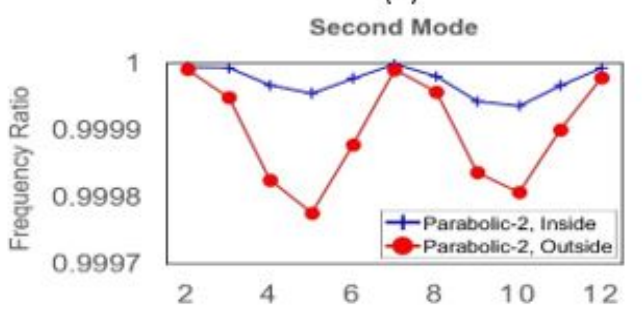

Position of Decomposition Along Curved Edge

(d)

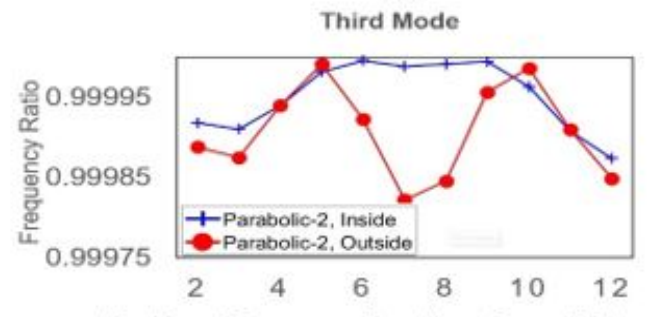

Position of Decomposition Along Curved Edge

(f)

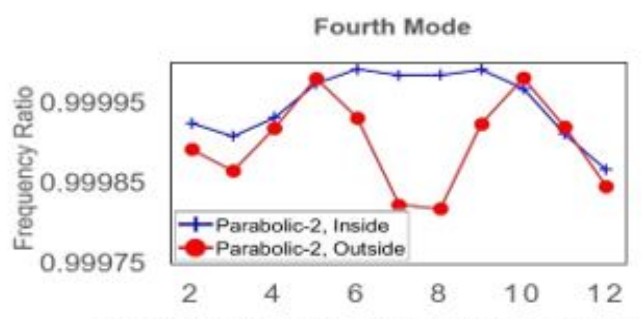

Position of Decomposition Along Curved Edge

(h)

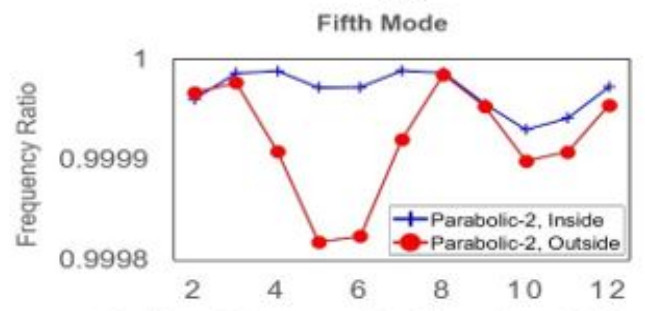

Position of Decomposition Along Curved Edge

(j)

Figure 10: Decomposition effects in parabolic curved plate. 
It can be interpreted from Figure 10 that the main reason that affects the frequency ratios of P2 structure is the relation of the location of the decomposition and the mode shapes of the structure. On the other hand, the effect of the stacking order takes place for the P1 structure. For instance, outside or inside decomposition located in the 12th row (see Figure 10 (a)) affected the first natural frequency ratio in different ways, which is a clear indicator of the fiber angles of the decomposed layers become a significant parameter on the frequency ratios. Similar behavior can be observed for all decomposition locations in Figure 10 (c) and various ones in Figures 10 (g) and (i).

Therefore, it can be concluded that the decompositions that occurred in different layers on the bending zones of the mode shape may have different effects on the natural frequency of the plate, depending on the modulus of elasticity, which has an active role according to the bending shape of the composite plate in the $x$ and $y$-axis.

It is concluded that the effect of the decomposition on the natural frequencies of curved structures is depended on the corresponding mode shapes, which are affected by the curvature geometry and the fiber orientation. As it is mentioned before, this is because of the variation of the fiber orientation and the decomposition level, which affects the rigidity of the structure. Therefore, decomposition effects on the structure are varied since the corresponding mode shapes' bending regions of the elliptic, circular, and parabolic curved structures are different.

\section{CONCLUSION}

Within the scope of the study, six-layered decomposed curved laminated composite plates having two different stacking orders are modeled by employing the Finite Element Method considering elliptic, circular, and parabolic curvatures. The effect of decomposition located along the curved edges on the free vibration response of the structure is obtained by adding decomposition separately to each row. In addition, the same analyses are conducted by modeling the decompositions in two different levels (outermost and the last two layers) in order to interpret the effect of decomposition on free vibration more clearly. The presented method, which can be applied in different directions, different stacking orders, and different geometric structures in future studies, will shed light on the effect of single or multiple damages on the dynamic characteristics of the system. The frequency ratios, which are obtained by proportioning the vibration responses of the damaged plates to the healthy plates pave the way for reverse engineering processes with the help of logic-based techniques such as machine learning. According to the generalized results, the following conclusions are drawn.

- $\quad$ CLPT represents different types of curves and stacking orders in thin composite plates accurately.

- The way that any decomposition affects the natural frequencies is directly correlated with the mode shapes of the curved structures.

- Decompositions occurred at the bending zones of the mode shapes of the structure affect the natural frequency values more than those located in other regions of the curved plate.

- $\quad$ The symmetric displacement behavior in mode shapes causes the symmetrical variation in the frequency ratios.

- The natural frequency values are affected less by the decompositions that occurred closer to the neutral axis of the curved structures.

- The decomposition occurrence in angle-ply and cross-ply laminates resulted in different effects on the natural frequency values due to the change in the value of the modulus of elasticity.

- The decomposition effects on the structure are varied since the corresponding mode shapes' bending regions of the elliptic, circular, and parabolic curved structures are different.

Author Contributions: Conceptualization, C Gonenli and O Das; Formal analysis, C Gonenli; Methodology, C Gonenli and O Das; Investigation, C Gonenli; Writing - original draft, C Gonenli; Writing - review \& editing, O Das.

Editor: Marco L. Bittencourt

\section{References}

An, H., Youn, B.D., Kim, H.S., (2021). Reliability-based design optimization of laminated composite structures under delamination and material property uncertainties. International Journal of Mechanical Sciences 205:106561. 
Arumugam, A.B., Rajamohan, V., (2017). Vibration analysis of rotating delaminated non-uniform composite plates. Aerospace Science and Technology 60:172-182.

Bathe, K.J. (2014). Finite element procedures, Prentice-Hall.

Chandrupatla, T.R., Belegundu, A.D. (2002). Introduction to Finite Elements in Engineering, Prentice Hall.

Dey, S., Karmakar, A., (2012). Finite element analysis of bending-stiff composite conical shells with multiple delamination. Journal of Mechanics of Materials and Structures 7(2):213-224.

Fazilati, J., (2017). Stability analysis of variable stiffness composite laminated plates with delamination using Spline-FSM. Latin American Journal of Solids and Structures 14(3):528-543.

Gay, D., Hoa, S.V., Tsai, S.W. (2003). Composite Materials Design and Applications, CRC Press.

Gonenli, C., Das, O., (2021). Effect of crack location on buckling and dynamic stability in plate frame structures. Journal of the Brazilian Society of Mechanical Sciences and Engineering 43(6):311.

Groh, R.M.J., Weaver, P.M., (2015). Static inconsistencies in certain axiomatic higher-order shear deformation theories for beams, plates and shells. Composite Structures 120:231-245.

He, Y., Xiao, Y., Su, Z., (2019). Effects of surface contact on the dynamic responses of delaminated composite plates. Composite Structures 229:111378.

Hirwani, C.K., Patil, R.K., Panda, S.K., Mahapatra, S.S., Mandal, S.K., Srivastava, L., Buragohain, M.K., (2016). Experimental and numerical analysis of free vibration of delaminated curved panel. Aerospace Science and Technology 54:353-370.

Juhász, Z., Szekrényes, A., (2020). An analytical solution for buckling and vibration of delaminated composite spherical shells. Thin-Walled Structures 148:106563.

Kamaloo, A., Jabbari, M., Tooski, M.Y., Javadi, M., (2019). Nonlinear free vibrations analysis of delaminated composite conical shells. International Journal of Structural Stability and Dynamics 20(1):2050010.

Kamaloo, A., Jabbari, M., Yarmohammad Tooski, M., Javadi, M., (2020). Nonlinear free vibration analysis of delaminated composite circular cylindrical shells. Journal of Vibration and Control 26(19-20):1697-1707.

Donnell, L.H. (1976). Beams, Plates, and Shells, McGraw-Hill Book Company.

Guha Niyogi, A., Laha, M.K., Sinha, P.K., (1999). Finite element vibration analysis of laminated composite folded plate structures. Shock and Vibration 6(5-6):273-283.

Ozturk, H., (2015). Vibration analysis of a pre-stressed laminated composite curved beam. Steel and Composite Structures 19(3):635-659.

Petyt, M. (2010). Introduction to Finite Element Vibration Analysis, Cambridge University Press.

Qin, B., Zhao, X., Liu, H., Yu, Y., Wang, Q., (2020). Free vibration analysis of curved laminated composite beams with different shapes, lamination schemes, and boundary conditions. Materials 13(4):1010.

Sahoo, S.S., Panda, S.K., Sen, D., (2016). Effect of delamination on static and dynamic behavior of laminated composite plate. AIAA Journal 54(8):2530-2544.

Tiwari, P., Barman, S.K., Maiti, D.K., Maity, D., (2019). Free vibration analysis of delaminated composite plate using 3d degenerated element. Journal of Aerospace Engineering 32(5):04019070.

Venkate Gowda, C., Rajanna, N., Udupa, N.G.S., (2017). Investigating the effects of delamination location and size on the vibration behaviour of laminated composite beams. Materials Today: Proceedings 4(10):10944-10951.

Wang, X., Wang, C., Schiavone, P., (2017). An interfacial arc crack in bonded dissimilar isotropic laminated plates. Journal of Mechanics of Materials and Structures 12(3):249-262. 